\title{
Apparent deficiency of mucosal vascular collagen type IV associated with angiodysplasia of the colon
}

\author{
D E Roskell, S C Biddolph, B F Warren
}

\begin{abstract}
Aims-To investigate the presence and distribution of vascular collagen type IV in colonic tissue in cases of angiodysplasia and age and sex matched controls.

Methods-Sections of colon from seven cases of colonic angiodysplasia and eight age and sex matched controls were examined for the presence of collagen type IV in vessels of the mucosa and submucosa. Immunohistochemical staining was performed on paraffin wax embedded sections, and the degree of vascular staining for each marker compared between mucosa and submucosa and between cases and controls. Staining for endothelial markers P-selectin and factor VIII was used to control for non-specific differences in immunostaining.
\end{abstract}

Results-In both the angiodysplastic tissues and approximately half the control tissues, staining for collagen type IV was considerably weaker in vessels in the mucosa than in the submucosa. In angiodysplasia, ectatic vessels in the mucosa appeared to contain less collagen type IV than similarly sized vessels in the submucosa, and perforating vessels appeared in many cases to lose staining at the level of the muscularis mucosae. No differences were found in staining intensity for the control endothelial markers between cases and controls.

Conclusions-The apparent relative deficiency of collagen type IV in the mucosal vessels in angiodysplasia may be related to their susceptibility to ectasia and haemorrhage. The finding of a similar deficiency in half of the control cases may reflect a population at risk of this relatively common condition.

(F Clin Pathol 1998;51:18-20)

Oxford University Nuffield Department of Pathology, John Radcliffe Hospital, Oxford OX3 9DU, UK D E Roskell

Department of Cellular Pathology, John Radcliffe Hospital S C Biddolph B F Warren

Correspondence to: Dr Roskell.

Accepted for publication 10 October 1997

\section{Keywords: collagen type IV; angiodysplasia; colon}

The term angiodysplasia was first used in 1974 to describe a condition of vascular ectasia of the bowel mucosa and submucosa that may lead to haemorrhage. ${ }^{1}$ It is most often found in the caecum and ascending colon of elderly people, and is probably the most common cause of gastrointestinal blood loss in this group, although it has been described in other sites and in very young children. ${ }^{1-3}$ There is no evidence that the vessels are neoplastic or that the lesions represent arterio-
The aetiology of angiodysplasia is unknown. venous malformations. Whether western dietary practices are important in the pathogenesis remains uncertain, although increased pressure in the colon caused by constipation has been suggested as a possible mechanism. ${ }^{4}$ Possible causes for ectasia of any vessel are the presence of an abnormally high intraluminal pressure and intrinsic weakness of the vessel wall. No such structural weakness has been described in angiodysplasia, nor is it known whether there are structural abnormalities of normal colonic vessels in those having or at risk of angiodysplasia.

Collagen type IV forms the basement membrane of vascular endothelium, and vessels deficient in this structural and thrombogenic substance might be susceptible to ectasia and haemorrhage such as is seen in angiodysplasia. In this study the presence of collagen type IV was investigated in mucosal and submucosal vessels of normal and angiodysplastic colonic tissue, and compared with the expression of the vascular markers P-selectin and factor VIII. Both control markers are present on the vascular endothelium and factor VIII is present on the subendothelial basement membrane. There is no reason why the expression of these markers should be altered in angiodysplasia. They were therefore considered suitable to control for non-specific variation in immunostaining, demonstrating that differences in staining were not simply a result of local factors in the tissue.

Table 1 Collagen type IV staining in mucosa and submucosa

\begin{tabular}{|c|c|c|c|}
\hline & Mucosa & Submucosa & $\begin{array}{l}\text { Relative } \\
\text { staining }\end{array}$ \\
\hline \multicolumn{4}{|l|}{ Cases } \\
\hline 1 & 1 & 3 & -2 \\
\hline 2 & 1 & 3 & -2 \\
\hline 3 & 1 & 2 & -1 \\
\hline 4 & 0 & 2 & -2 \\
\hline 5 & 0 & 2 & -2 \\
\hline 6 & 1 & 3 & -2 \\
\hline 7 & 0 & 3 & -3 \\
\hline \multicolumn{4}{|c|}{ Mean relative mucosal vascular collagen type IV } \\
\hline \multicolumn{4}{|l|}{ Controls } \\
\hline A & 0 & 2 & -2 \\
\hline B & 2 & 2 & 0 \\
\hline $\mathrm{C}$ & 1 & 3 & -2 \\
\hline D & 1 & 3 & -2 \\
\hline $\mathrm{E}$ & 2 & 2 & 0 \\
\hline $\mathrm{F}$ & 2 & 2 & 0 \\
\hline G & 0 & 2 & -2 \\
\hline $\mathrm{H}$ & 1 & 1 & 0 \\
\hline \multicolumn{3}{|c|}{$\begin{array}{l}\text { Mean relative mucosal vascular collagen type IV } \\
\text { staining }\end{array}$} & -1 \\
\hline
\end{tabular}


Table 2 Factor VIII staining in mucosa and submucosa

\begin{tabular}{llll}
\hline & Mucosa & Submucosa & $\begin{array}{l}\text { Relative } \\
\text { staining }\end{array}$ \\
\hline Cases & & & \\
1 & 2 & 2 & 0 \\
2 & 3 & 3 & 0 \\
3 & 2 & 2 & 0 \\
4 & 2 & 2 & 0 \\
5 & 3 & 3 & 0 \\
6 & 3 & 3 & 0 \\
7 & 2 & 2 & 0 \\
Mean relative & mucosal vascular collagen type IV & \\
staining & & & 0 \\
& & & \\
Controls & 1 & 1 & 0 \\
A & 2 & 2 & 0 \\
B & 1 & 1 & 0 \\
C & 3 & 3 & 0 \\
D & 2 & 2 & 0 \\
E & 3 & 3 & 0 \\
F & 2 & 2 & 0 \\
G & 2 & 2 & 0 \\
H & & & \\
Mean relative mucosal vascular collagen type IV & \\
staining & & & \\
\hline
\end{tabular}

\section{Patients and methods}

Routinely prepared, formalin fixed, paraffin wax embedded sections were processed from seven cases of colonic angiodysplasia and eight age and sex matched controls who had undergone colectomy because of carcinoma. The angiodysplasia patients were three men aged 66,68 , and 79 years, and four women aged $77,80,82$, and 83 years, respectively. The control group comprised four men aged 69, 70, 73 , and 81 years, and four women aged 78,81 , 83 and 84 years, respectively. Immunohistochemical characterisation of the blood vessels in the mucosa and submucosa was done using
Table 3 P selectin staining in mucosa and submucosa

\begin{tabular}{lccc}
\hline & Mucosa & Submucosa & $\begin{array}{c}\text { Relative } \\
\text { staining }\end{array}$ \\
\hline Cases & 1 & 1 & \\
1 & 1 & 1 & 0 \\
2 & 1 & 1 & 0 \\
3 & 1 & 1 & 0 \\
4 & $\mathrm{U}$ & $\mathrm{U}$ & 0 \\
5 & 1 & 1 & 0 \\
6 & 2 & 2 & 0 \\
7 & & & 0 \\
Mean relative mucosal vascular collagen type IV & \\
staining & & & 0 \\
Controls & & & \\
A & 1 & 1 & 0 \\
B & 2 & 3 & -1 \\
C & 2 & 0 \\
D & 1 & 1 & 0 \\
E & 2 & 2 & 0 \\
F & 2 & 2 & 0 \\
G & U & U & 0 \\
H & 1 & 1 & -0.14 \\
Mean relative mucosal vascular collagen type IV & \\
staining & & &
\end{tabular}

the alkaline phosphatase antialkaline phosphatase technique with a fast red chromogen. ${ }^{5}$ Commercially available primary monoclonal antibodies to P-selectin (JC70; Dako, High Wycombe, Bucks, UK), factor VIII, and collagen type IV were used.

Staining intensity was assessed in both the mucosa and submucosa using a simple scoring system of $0,1,2$, and 3 , representing from none to very strong staining. The submucosal staining score was subtracted from the mucosal score to give a relative intensity for each case. Thus a relative score of 0 was recorded if there

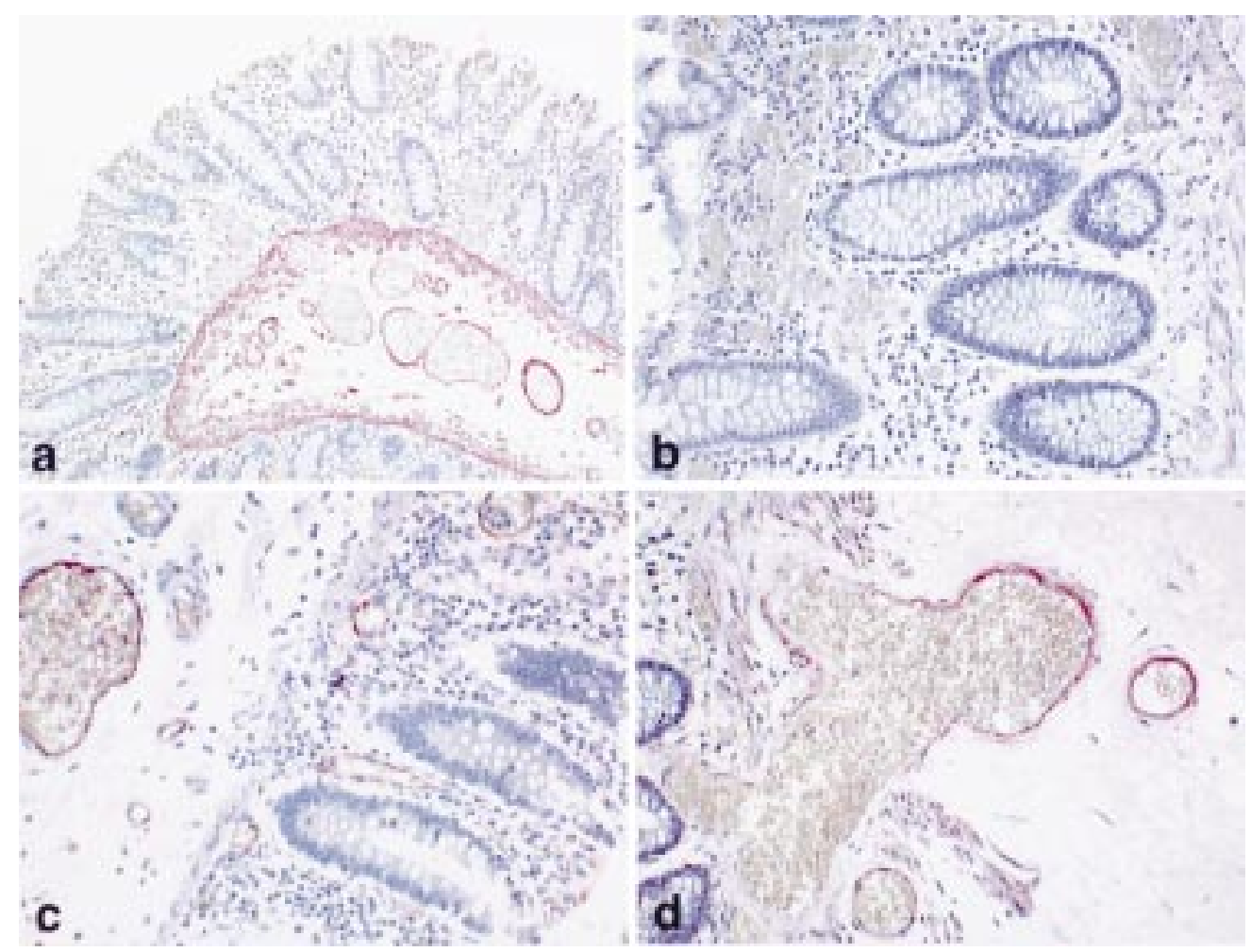

Figure 1 Typical case of angiodysplasia stained for collagen type IV demonstrating strong staining of vessels in the submucosa and absence in the mucosal vessels (original magnifications $(a) \times 40(b) \times 100)$ (c) Typical staining for $P$-selectin showing staining of both mucosal and submucosal vessels (original magnification $\times 100$ ). (d) High power of angiodysplasia showing apparent loss of collagen type IV in a vessel passing through the muscularis mucosae (original magnification $\times 100$ ). 
was no difference in mucosal and submucosal vascular staining, while a score of +3 indicated very strong staining in the mucosa with none in the submucosa. A score of -2 indicated considerably less staining in the mucosa than in the submucosa.

\section{Results}

Tables 1-3 show the staining intensities and relative staining for the various markers. In angiodysplasia, ectatic vessels in the mucosa appeared to contain less collagen type IV than similarly sized vessels in the submucosa, and perforating vessels appeared in many cases to lose collagen type IV at the level of the muscularis mucosae (fig 1). This relative deficiency of collagen type IV was seen in small, apparently normal mucosal vessels in angiodysplasia cases as well as in the ectatic vessels themselves. Similar but lesser changes were seen in half of the age matched controls.

No differences were found in staining intensity for the control vascular markers P-selectin and factor VIII related antigen.

\section{Discussion}

The aetiology of angiodysplasia of the colon remains uncertain. No differences in collagen type IV were found to be specific for the condition, although all of the cases appeared to contain less collagen type IV in mucosal vessels than in the submucosa, a similar reduction being seen in half of the age matched controls. The reduction in collagen type IV staining in the mucosa is unlikely to be artefactual as no similar reductions were seen with the control vascular markers. Because collagen type IV has a structural and thrombogenic role in the vessel wall, it is reasonable to suggest that this pronounced lack of collagen type IV in mucosal vessels of patients with angiodysplasia may have led to weakness, ectasia, and haemorrhage.

If a loss of collagen type IV is involved in the pathogenesis of angiodysplasia, the demonstration of its loss in half of the control group needs to be explained. One possibility is that it is reduced in half the population, it is unrelated to angiodysplasia, and our angiodysplasia cases happened by chance to all fall into the group with reduced mucosal vascular collagen type IV. More attractive is the idea that loss of collagen type IV from mucosal vessels is a prerequisite for symptomatic angiodysplasia. The "normal" controls with loss of vascular collagen type IV could have asymptomatic angiodysplasia. However, the incidence of asymptomatic angiodysplasia estimated from colonoscopic studies is much lower (approximately $1 \%^{6}$ ), and a more reasonable suggestion is that the finding of decreased collagen type IV in mucosal vessels of half of the morphologically normal age matched controls might identify a population at risk of the condition.

If this at risk population can be identified, it is probably too large to be of much clinical use in establishing the cause of gastrointestinal blood loss. However, it might be worthwhile to investigate putative factors in the aetiology of angiodysplasia, such as diet and age, to establish whether there is an association with loss of mucosal vascular collagen IV with or without the development of symptomatic angiodysplasia.

1 Rees HC, Wright NA. Angiodysplasia of the colon. In: Anthony PP, McSween RNM, eds. Recent advances in histopathology. Vol 12. Edinburgh: Churchill Livingstone 1984:178-88.

2 de la Torre Mondragon L, Vargas-Gomez MA, MoraTiscarreno MA, et al. Angiodysplasia of the colon in children. I Pediatr Surg 1995;30:72-5.

3 Gupta N, Longo WE, Vernava AM. Angiodysplasia of the lower gastrointestinal tract: an entity readily diagnosed by colonoscopy and primarily managed nonoperatively. Dis Colonoscopy and primarily man 1995;38:979-82.

Colon Rectum 1995;38:979-82.
4 Whitehead R, Gratama S. Vascular disorders of the large intestine. In: Whitehead R, ed. Gastrointestinal and oesophageal pathology. 2nd edn. Edinburgh: Churchill Livingstone 1995:687-709.

5 Cordell JL, Falini B, Erber WN, et al. Immunoenzyme labelling of monoclonal antibodies using immune complexes of alkaline phosphatase and monoclonal antialkaline phosphatase (APAAP) complexes. $\mathcal{F}$ Histochem Cytochem 1984;32:219-29.

6 Foutch PG, Rex DK, Lieberman DA. Prevalence and natural history of colonic angiodysplasia among healthy asymptomatic people. Am f Gastroenterol 1995;90:564-7. 